\title{
Appropriate Level of Protection: \\ A New Zealand Perspective ${ }^{1}$
}

\author{
HUGH R. BIGSBY \\ Commerce Division \\ P.O. Box 84 \\ Lincoln University \\ Canterbury \\ New Zealand
}

\begin{abstract}
The paper presents a model for quantifying quarantine-related trade barriers by combining the two basic components of pest risk assessment, probability of establishment and economic effects, into a single management framework, Iso-Risk. The model provides a systematic and objective basis for defining and measuring acceptable risk and for justifying quarantine actions relative to acceptable risk.
\end{abstract}

\section{Introduction}

One of the outcomes of the Uruguay Round of the General Agreement on Tariffs and Trade (GATT) was the provision for reductions in a range of trade barriers. In particular, certain types of barriers, such as tariffs, export subsidies, embargoes, import bans, quotas, supply management regimes, domestic price supports, licensing and exchange controls, were able to be dealt with by converting them into "tariffequivalent' levels of protection through a system of 'tariffication'. The key success of this approach was that different 'quantifiable' trade barriers could then be compared, reduced or negotiated in a common framework of tariffs. What remained to be resolved after the Uruguay Round were a range of trade barriers that were largely non-quantifiable in terms of tariff-equivalent levels of protection. These barriers, termed 'Technical Barriers to Trade' (TBT), included rules and standards directed at health, safety or the environment.

A key feature of TBTs, which differentiates them from quantifiable trade barriers, is that they are not specifically targeted at trade or production issues. Under GATT rules, countries are "generally allowed" to adopt health, safety or environmental policies which take precedence over other rules. The caveat to this, however, is that these policies are only allowed as long as the purpose of the policy or standard is to meet a legitimate domestic objective, and as long as domestic and foreign producers are treated in the same manner.

Among the most prevalent types of TBT's are those that deal with concerns about human, animal and or plant health (Hillman 1978, 1991). With the reduction in quantifiable barriers to trade, concern has been raised that countries will turn to TBT's as a way of blocking imports rather than just meeting legitimate sanitary and phytosanitary concerns (Ndayisenga and Kinsey 1994). This concern has led to major efforts internationally to ensure that sanitary and phytosanitary measures do not evolve as major trade barriers.

Under the World Trade Organisation (WTO), TBT's related to animal and plant health issues are dealt with under the Sanitary and Phytosanitary (SPS) Agreement. Under the umbrella of the SPS Agreement, the International Plant Protection Convention (IPPC) has produced standards for determining the Appropriate Level of Protection (ALP), or justified quarantine measures, for plants (FAO 1996). The major problem faced by the IPPC is the lack of a system that can convert diverse technical or scientific barriers related to plant health into a common framework which would allow comparison of quarantine

1 Bigsby, H. R. (2001). Appropriate level of protection: a New Zealand perspective. In, The Economics of Quarantine and the SPS Agreement, edited by K. Anderson, C.F.McRae and D. Wilson, Biosecurity Australia, Canberra and Centre for International Economic Studies, University Adelaide. Pp 141-163. 
measures within a trade, or economic, forum. A common theme of the activity of the IPPC is a need to develop systems that will provide a measure of the Appropriate Level of Protection (ALP). This in turn will show whether health or phytosanitary standards are being imposed in a way that is consistent with both internal and external standards.

Another important change with the Uruguay Round has been a move to focus on risk assessment and management with an overall objective of minimising negative trade impacts (Papasolomontos 1993). This is a considerable departure from past practice in the quarantine area. Historically SPS has been the domain of scientists and the key criteria for applying trade barriers has been an assessment of probability of occurrence (Smith 1993, Patterson 1990). This is an objective, but one-sided application of standards in a trading environment. Under the Uruguay Round, risk assessment now requires consideration of economic consequences as well as probability of occurrence. In addition, risk management now requires the consideration of trade-offs in probability of establishment and economic consequences, and in the context of choosing the least trade-distorting path.

This paper presents a risk analysis system, the Iso-Risk framework, which addresses the problems created by TBT's in the post-Uruguay Round environment. The Iso-Risk framework does this by combining the key elements of risk analysis, probability of occurrence and economic impact, into a single analytical system, providing a quantifiable measure of the level of protection associated with a quarantine measure.

\section{Iso-Risk Framework}

The major problem presented by TBT's is the lack of a system which can convert diverse technical barriers related to plant or animal health into a common framework which allows for comparison in a trade forum. In other words, what kind of a measure will adequately combine the key features of risk analysis, risk of introduction and economic consequences, in a way which facilitates comparison and negotiation? The greatest need is to convert barriers to values that are common in a trade environment, typically currency measures. A way for eliciting the value of a TBT is by measuring implicit or explicit economic effects that are created by the barrier. This could be done in the context of measuring the value of events related to a TBT. Examples of this could include measuring the additional costs associated with compliance with a regulation, new labelling or packaging, or reducing residues. This could also be done in the context of measuring the value of an outcome without a technical barrier in place. In this case the consequences of an economic impact such as a pest infestation could be measured.

An important component of assessing risk or levels of protection is a methodology that uses both economic effects and probability of introduction to manage risk (FAO 1996). Although the FAO's draft standards do not specify how to combine economic effects and probability of introduction, the implication is that they should be considered together to measure 'Pest Risk'.

A common way for these two factors to be combined is to calculate Pest Risk as,

$$
\text { Pest Risk }=\text { Economic Effect } \mathrm{x} \text { Probability of Introduction }
$$

Use of both the probability and consequences of a particular event to express risk appears in many areas of risk analysis (Kaplan and Garrick 1981, Cohrssen and Covello 1989, Miller et al. 1993, Ministry of Health 1996). The framework discussed here follows this approach and comes from discussions during the development of the draft Pest Risk Analysis Standards by the IPPC working group (Orr 1995) and has been further developed in New Zealand (Bigsby 1996, Bigsby and Crequer 1998, Bigsby and Whyte 1998, 2000).

Calculated this way, Pest Risk represents the expected value of the economic effect of pest introduction during the time period for which the probability of introduction has been assessed. If a quarantine authority used this definition for Pest Risk, risk management options would be considered in the context of some benchmark or acceptable level of Pest Risk (equivalent to ALP) and the need to alter the 
probability of introduction or the economic consequences of establishment. A critical component is the establishment of a benchmark level of acceptable pest risk, so that subsequent management strategies can be systematically evaluated against the benchmark.

\section{Pest Versus Commodity Risk Assessment}

Many quarantine risk assessments focus on the risk associated with a particular pest. However, trade restrictions and most pre-entry quarantine measures are directed at entire commodities rather than particular pests. In this paper, 'commodity' refers to a specific product and country/pathway combination. The important distinction here is that commodities with more types of pests will represent a greater risk, per unit, than commodities with fewer types of pests. A purely pest-based analytical approach, while useful for some types of analyses, such as categorising pests into quarantine and nonquarantine, may not give a measure of the overall risk associated with a commodity.

Commodity-based risk assessments, such as those produced by the USDA (USDA 1996) rely on assessments of each pest associated with a commodity. Similarly, the appropriate level of protection can be defined for a commodity by considering the appropriate levels of protection for each individual pest of the commodity. Given the distinction between the two different approaches, risk assessment for individual pests and commodities will be discussed separately.

\section{Pest Evaluation}

The Iso-Risk framework for individual pests is illustrated in Figure 1. Pest 1, with an economic impact of $\mathrm{EI}_{1}$ and a probability of establishment of $\mathrm{r}_{1}$, has a pest risk of $\mathrm{PR}_{1}{ }^{2}$, where,

$$
\mathrm{PR}_{1}=\mathrm{EI}_{1} \times \mathrm{r}_{1}
$$

Pest 2 has an economic impact of $\mathrm{EI}_{2}$ and a probability of introduction of $\mathrm{r}_{2}$. As can be seen in Figure 1, different pests, having different potential economic consequences and probability of introduction, may still share the same value of pest risk. Both $\mathrm{PR}_{1}$ and $\mathrm{PR}_{2}$ lie on the same line where all combinations of $\left(\mathrm{EI}_{\mathrm{i}} \times \mathrm{r}_{\mathrm{i}}\right)$ have the same value (hence, the 'iso-risk' line). Note that the iso-risk line is straight only when both the $\mathrm{x}$ and $\mathrm{y}$ axes are plotted with logarithmic scales.

A key requirement for carrying out risk assessment, or determining entry conditions, is a pre-determined benchmark level of pest risk, or ALP, from which to base decisions. In Figure 1 there will be an infinite number of iso-risk lines representing different levels of Pest Risk, with higher iso-risk lines indicating higher Pest Risk. Iso-risk lines allow pests to be compared to each other, and compared to a particular acceptable level of Pest Risk. This ability to compare in turn provides the basis for determining appropriate actions. In particular, the result of pest risk management should be a Pest Risk that does not exceed the ALP, with a reasonable level of confidence. In the context of Figure 1, since all points on an iso-risk line have the same expected value,

\section{The ALP represents the highest iso-risk line that will be accepted by a quarantine authority.}

2 Pest Risk is depicted in Figure 1 as a point estimate or single value. This is done for purposes of illustration in developing the general methodology in this chapter. In practice, there would be a problem in providing only a point estimate because it gives no quantitative picture of the uncertainty surrounding either the probability of establishment or economic impact values used in the pest risk estimate. This means that there is no information on whether a particular estimate represents the most likely value, or one of a host of equally likely values over a wide range [for example, Cohrssen and Covello 1989]. Since pest risk is actually based on a probability distribution for both risk of introduction and economic impact, rather than being a point estimate, a plot of pest risk would be an area. Given a distribution of outcomes, a decision maker would be in a position to make a better-informed assessment of the appropriate management actions for a particular pest than with only a point estimate. 
Figure 1

Iso-Risk Framework

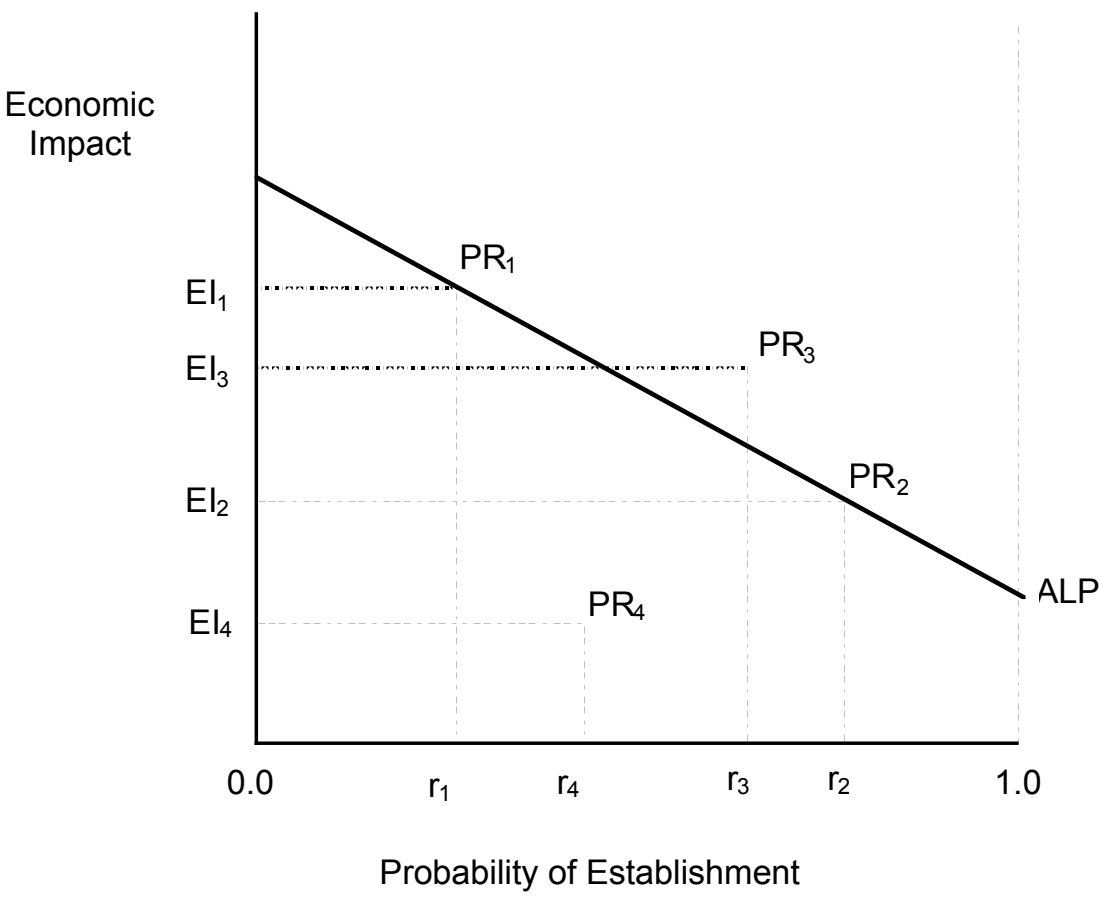

Given this definition, individual pests can be evaluated against an ALP. If the Pest Risk of a particular pest is greater than the ALP, actions should be taken to reduce Pest Risk to the ALP. For example, if the iso-risk line in Figure 1 has been determined to be the ALP, a pest with a Pest Risk of PR 3 would be subject to actions to reduce the risk to acceptable levels. The pest corresponding to $\mathrm{PR}_{4}$ falls within acceptable limits, and requires no additional quarantine actions.

\section{Commodity-based Risk Assessment}

The pest risk of a commodity (PRC) can be considered as the cumulative expected value of all the associated pests for that commodity. If PRC is the expected value of pest risk for a commodity, then,

$$
\operatorname{PRC}=\sum_{i=1}^{n}\left(R_{i} \times E_{i}\right)
$$

where $\mathrm{R}_{\mathrm{i}}$ is the probability of establishment of pest $\mathrm{i}, \mathrm{E}_{\mathrm{i}}$ is the economic impact of pest $\mathrm{i}$, and $\mathrm{n}$ is the number of pests associated with the commodity. Since PRC is the sum of a number of individual Pest Risks, it can take any value from 0 to $\infty$, as is shown in Figure 2.

With this approach, a quarantine authority could consider commodities having similar values of PRC, regardless of the number or type of pests involved, with the same level of concern. A benchmark ALP can also be defined for commodities as follows:

The ALP is the highest value of commodity pest risk that will be accepted by a quarantine authority. 
In Figure 2, the ALP would represent a cut-off point on the axis. Appropriate entry conditions would ensure that the commodity risk does not exceed the ALP with a reasonable level of confidence.

Figure 2

Commodity Pest Risk (PRC)

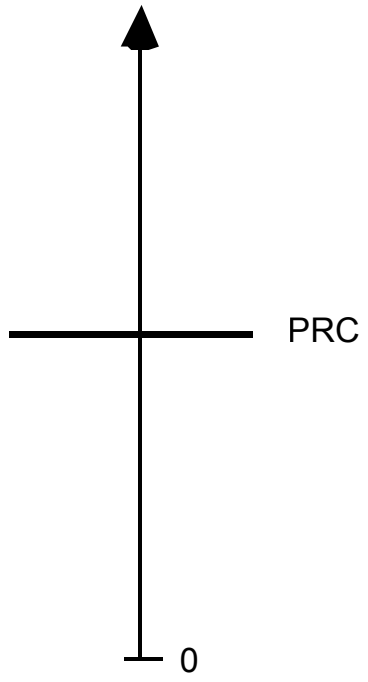

\section{Application of the Iso-Risk Framework}

The discussion thus far has been based on what is effectively a quantitative assessment of risk, or one that consists of a continuous set of numeric values from which to estimate ALP (the iso-risk line). Some quarantine agencies have developed qualitative rather than quantitative guidelines for pest risk assessment. For example, APHIS-PPQ in the U.S. has produced a set of guidelines that identify pest risk in terms of high, medium or low (USDA 1996). The difference between the two approaches is that a measure of pest risk based qualitative values results in discrete, categorical values.

As long as pest risk is expressed in terms of probability of introduction and economic impact though, either approach can be expressed in terms of the iso-risk model. Applications of both the quantitative and qualitative approaches to risk assessment using the Iso-Risk framework are provided in the following sections.

\section{Quantitative Risk Assessment}

Whyte (1998) provides an application of the Iso-Risk Framework to Mediterranean fruit fly, Ceratitis capitata. Risk assessments for the Mediterranean fruit fly were used to examine risk assessment and management for pests entering New Zealand by both commercial and non-commercial pathways. The risk assessments showed that with no quarantine measures the annual probability of introduction was effectively 1.0. The economic consequences to producers of Mediterranean fruit fly establishment on an annual basis, after population stabilisation, were predicted to be $\$ 100$ - \$130 million (Whyte 1998). Using a midpoint for economic impact of $\$ 121$ million, the initial Pest Risk is $\$ 121$ million. This is shown as Point 1 in Figure 4, lying on an iso-risk line for \$121 million.

For commercial pathways, the introduction of a probit 9 treatment or area freedom reduces the annual probability of introduction to about 0.0017 and the Pest Risk becomes $\$ 206,000$. This is shown as the horizontal shift of Pest Risk from the initial position to the new probability of introduction at Point 2 in Figure 4 lying on an iso-risk line for $\$ 206,000$. For non-commercial pathways, the introduction of $\mathrm{x}$-ray machines to international airports has reduced the annual probability of introduction to about 0.02 and the Pest risk becomes $\$ 2.42$ million. This is shown as the horizontal shift of Pest Risk from the initial 
position to the new probability of introduction at Point 3 in Figure 4 lying on an iso-risk line for $\$ 2.42$ million.

Figure 4

Quantitative Risk Assessment

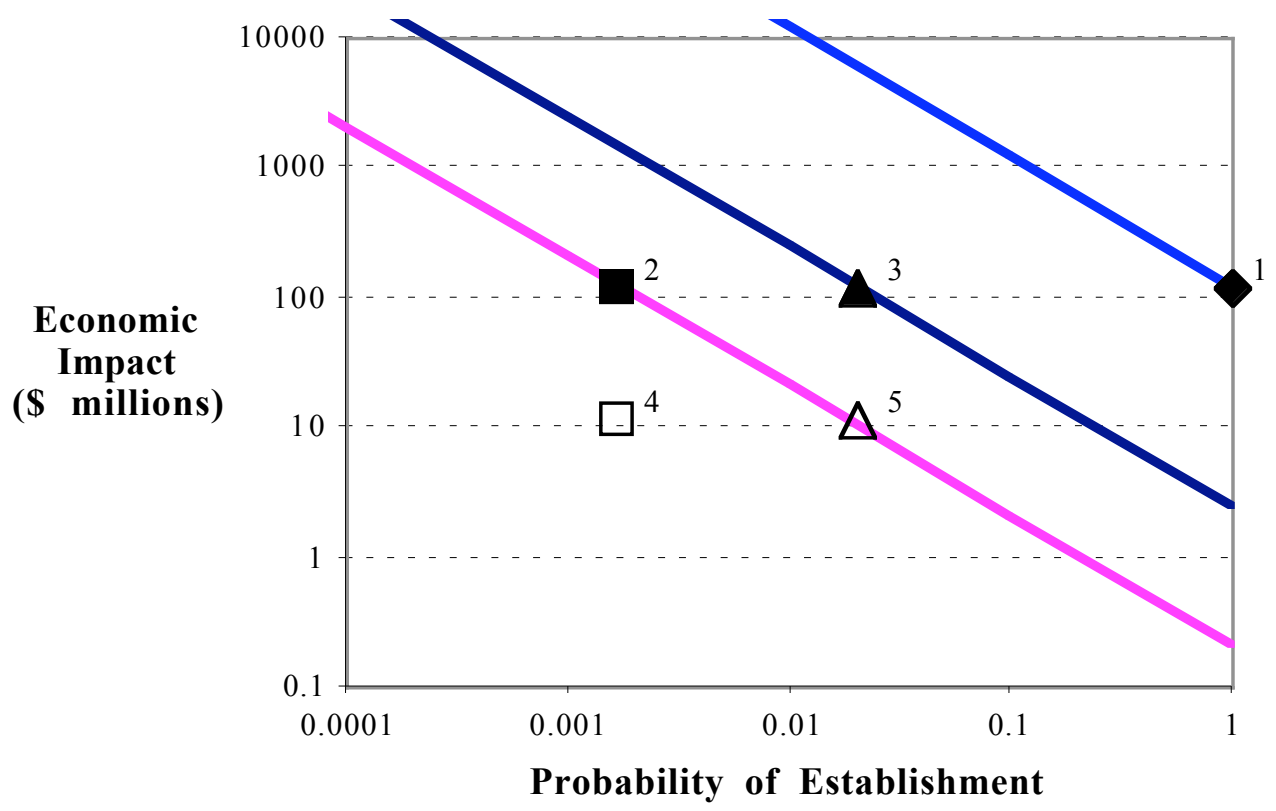

Note: Both axes are plotted in log scales

While it might be tempting to imply from this limited example that New Zealand's ALP lies between \$0.206 and \$2.42 million, additional measures to limit the risk of Mediterranean fruit fly establishment are also employed. In addition to the probit 9 treatment for commercial pathways and the use of x-ray machines for non-commercial pathways, a detection and response system for Mediterranean fruit fly is also provided. A surveillance system consisting of trimedlure trapping has been put in place that allows for rapid detection and response to an establishment.

Using the cost of the 1996-97 Mediterranean fruit fly response of approximately $\$ 5.3$ million and assuming a five percent chance that an eradication attempt would fail even with the early warning system (Bigsby and Whyte 1998, Whyte 1998), the effect of this additional risk management system can be estimated. The early warning and eradication programme further reduces the probability for either pathway that there would be an establishment that had an economic impact. In terms of the iso-risk model, this system means that the expected annual economic impacts would be reduced to approximately $\$ 11.35$ million for the either pathway. Given that the commercial pathway still has a probability of 0.0017 for an establishment, the new Pest Risk is $\$ 19,300$, shown as Point 4 in Figure 4. For the noncommercial pathway the probability of establishment is still 0.02 so the new Pest Risk is $\$ 227,000$, shown as Point 5 in Figure 4. This would reduce the implied ALP based on this example to somewhere between $\$ 19,300$ and $\$ 227,000$.

\section{Qualitative Measures of Pest Risk}

As was mentioned previously, some agencies, such as APHIS-PPQ in the U.S., have developed qualitative guidelines for pest risk assessment. This results in discrete, categorical values to express Pest Risk. However, as long as pest risk is expressed in terms of probability of introduction and economic impact, qualitative values can be adapted to fit the iso-risk model. 
An example of how this adaptation can work is the U.S. pest risk assessment system (USDA 1996). Similar to the iso-risk framework, the U.S. guidelines provide the basis for ranking pest risk based on potential consequence of introduction and likelihood of introduction. Rather than a dollar value though, potential consequence of introduction is comprised of five "risk elements". The risk elements, climatehost interaction, host range, dispersal potential, economic impact and environmental impact, are each given a score of high, medium or, with a score of high given 3 points, medium 2 points and low 1 point. A cumulative score for the five risk elements is then calculated. A similar process is carried out for likelihood of introduction, but with only two risk elements, quantity of commodity imported and pest opportunity (survival and access to suitable habitat and hosts). In each case, the cumulative risk element score results in a new risk rating of high, medium or low, again with the same corresponding risk scores for each as before. In the last step of the process, likelihood of introduction and consequences of introduction are combined by calculating a cumulative value based on the risk score for each component. The cumulative value provides a pest risk potential, rated high, medium or low depending on the score.

If the raw risk scores for likelihood of introduction and consequences of introduction are used rather than the high, medium and low ranking, this system of scoring can be adapted to the iso-risk framework. As is shown in Figure 5 the APHIS-PPQ system allows for a maximum score for consequence of introduction of 15 and for likelihood of introduction of 18. Based on how the APHIS-PPQ guidelines translate combinations of cumulative risk element scores for likelihood of introduction and consequences of introduction into risk management options, Figure 5 can also be separated into risk management zones (Bigsby and Whyte 1998).

Figure 5

Qualitative Risk Assessment

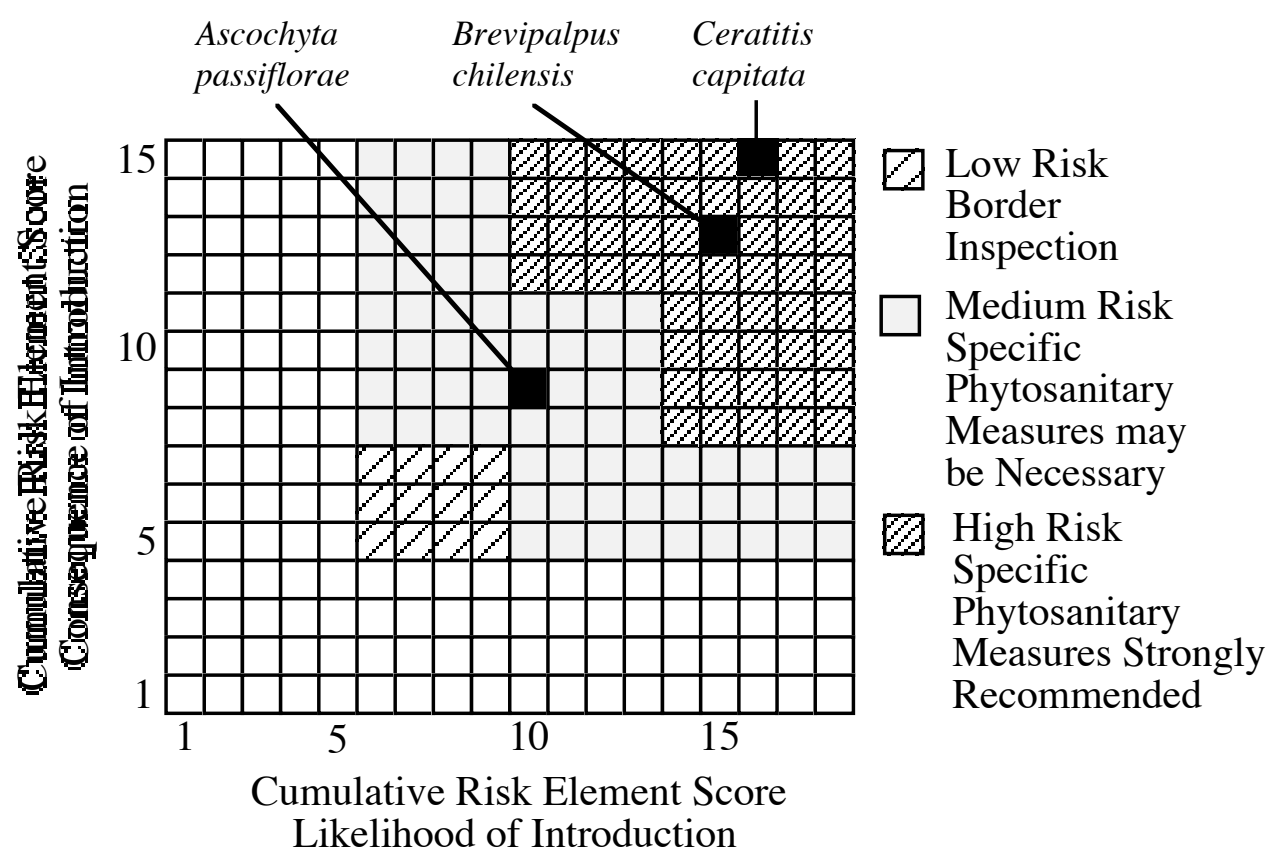

If Figure 5 is put in the context of Figure 1, the similarities in terms of iso-risk and management options can be seen. With the cruder measures of economic impacts and probability of establishment, the iso-risk line becomes less distinct, but none the less can be defined in terms of combinations that represent acceptable levels of risk. The major difference from Figure 1 is that there is now a zone of risk that creates a wide bound in which the actual, but undefined, iso-risk line lies. This wide bound arises due to the wide range of values from the cumulative risk element scores for either the consequence of introduction or the likelihood of introduction which are collapsed in the U.S. system. In Figure 5, the iso- 
risk line/zone is defined by the shaded cells. Pests with risk values in the shaded cells represent unacceptable levels of risk for which quarantine measures might be undertaken.

An example of how a qualitative risk assessment could be adapted is a pest risk assessment for the import of Purple Passion Fruit (Passiflora edulis) from Chile to the U.S. (Firko and Podleckis 1996). Three potential pests associated with Purple Passionfruit were identified Ascochyta passiflorae, Brevipalpus chilensis, and Ceratitis capitata. Based on information provided by Firko and Podleckis (1996), the cumulative risk element scores for consequence of introduction and likelihood of introduction respectively are 9 and 10 for Ascochyta passiflorae, 13 and 15 for Brevipalpus chilensis, and 15 and 16 for Ceratitis capitata. These values are plotted in Figure 5.

Since Firko and Podleckis (1996) is a qualitative risk assessment only, they made no attempt to suggest risk management options or to fit them within the risk assessment framework. The expectation would be that risk management would modify the likelihood of introduction. This would move the combined cumulative risk for each of the potential pests horizontally to the left far enough to provide an acceptable level of risk.

One complicating factor with the use of qualitative inputs is that the categorical values are derived from a range of factors (risk elements) that have no common denominator. This means that "expected value" from combining an economic impact and a probability is not applicable here. Risk scores for consequence of introduction and likelihood of introduction can still be multiplied, but the resulting is something different than an expected value. The combined risk rating can still be used to rank a pest and to form an iso-risk line, but only relative to this risk rating system.

\section{Methodological Issues}

The use of economic effects and probability of occurrence or introduction to calculate an expected value, while relatively straightforward, can only be done after considering what constitutes an appropriate measure of economic effects and probability of introduction. The iso-risk model described so far has been based on a broadly defined measure of economic impacts. There are however, a number of potential economic impacts that could be included in an assessment, ranging from potential pest-related damage to the benefits of lower world prices for commodities. This in turn raises the question about what the objective of the assessment should be, and in particular, whether it is designed to provide a safety standard or welfare maximisation. In a similar way, a broadly specified risk of introduction masks the fact that probability of introduction is related to the volume of trade. This raises a question of whether the risk assessment process is attempting to provide an envelope to risk or whether it will let risk fluctuate with trade levels.

\section{Safety Standard versus Welfare Maximisation}

One of the issues to be considered is what should be included under economic effects in the model. Generally speaking, there are two broad perspectives on what should be included, each related to the underlying objective of the analysis. One perspective is that the objective of the analysis should be safety and that the development of a safety standard is a key outcome. The other perspective is that the objective of the analysis should be welfare maximisation. The importance of which of these perspectives is chosen is that it will influence which economic effects are measured and how they will be interpreted.

Before comparing these two perspectives, it is useful to look generally at how economic effects might be measured. In the context of pest risk assessment, at a basic level economic effects might be considered as either direct or indirect pest effects. Direct pest effects would be direct impacts of a pest on a host plant, and would cover host-specific impacts like yield loss or mortality. Indirect pest effects would be nonhost specific impacts. These would be general effects that are created by the presence of a pest, but not specific to the pest-host dynamic, including public health issues, restrictions on traffic flow, key ecosystem function compromised, research requirements, market access problems, and tourism. At a 
broader level, economic effects could extend to the market for the products covered by the analysis, expanding the analysis to include consumers.

The types of economic analysis that are relevant are linked to the scope or level of economic activity that is being measured. Following the FAO (1996) guidelines, economic analysis can be grouped into partial budget, partial equilibrium, general equilibrium and non-commercial/environmental analyses. Partial budget analysis is the narrowest in scope and deals mainly with changes to the profits of individual producers. Examples of this approach in quarantine risk assessment include Cowley et al. (1993), DPIE (1997), Whyte et al. (1995), Whyte and Cowley (1996), and Whyte et al. (1998). Partial equilibrium analysis is wider in scope than partial budget, dealing with a production sector as whole rather than individual producers. Examples of this approach in quarantine risk assessment include James and Anderson (1998), Roberts et al (1999) and USDA 1991). General equilibrium analysis extends the analysis to encompass an entire economy, and allowing the effects on wages, exchange rates and national welfare to be measured. These approaches form a progression of analytical opportunities that are available as the scope of an impact increases, moving from the narrowest to the widest in scope.

With a safety perspective and safety standard objective, the key motivation is minimising the potential for negative outcomes. The focus on negative outcomes in turn means that the economic analysis would be limited to the measurement of the negative impacts of a potential pest on an economy. This limits what is considered in an economic analysis rather than the level of the analysis. Since economic effects are linked to how a pest or disease manifests itself, the extent of physical effects a pest has on a host, the number of potential hosts, the effect of existing control measures, and the effect of existing management practices becomes important. In terms of the Iso-Risk framework, the safety standard objective means reducing potential negative effects towards an identified ALP. An important outcome of this approach is that the benchmark for decisions (safety standard or ALP) is constant across all decisions.

With a welfare maximisation perspective, the objective becomes one of welfare maximisation rather than attempting to meet a particular standard. Benefits to consumers, as well as negative impacts on producers would be considered in the analysis. A possible outcome of this approach is that quarantine measures would be rejected because consumer benefits outweighed producer costs. The problem with this perspective as tool for setting quarantine policy is that the decision on how any particular commodity would be treated would depend on the nature of the market for the commodity.

The Mediterranean fruit fly example discussed earlier provides an example of the issues raised in adopting a welfare-maximising approach rather than a safety approach to determining quarantine standards. The two pathways in this example could instead be two different commodities that carried the same pest but presented different risks. Using the safety approach, both commodities would be subject to sufficient and possibly different quarantine measures to reduce their Pest Risk to a common acceptable level. Under the welfare-maximising approach, if the commodity which had a high probability of introducing the pest also provided a higher net welfare gain to consumers if it was unregulated rather than regulated, the commodity would be unregulated. This would compromise the effect of the quarantine measure imposed on the other commodity and raise questions about the justification of the measure.

\section{Global or Individual Appropriate Level of Protection}

One factor that must be considered is the 'level' of risk that will be considered. The choices of level of risk for a regulator are (1) whether there is a desire to fix total risk associated with all trade within a given period, or (2) whether there is a desire to fix the risk associated with a particular commodity or pest. A key factor in this decision is the correlation between the likelihood of introduction of a pest and the volume of trade that is carried out.

In the case of fixing total risk associated with all trade, the regulator determines a maximum expected value that will be accepted, aggregated over all commodities imported during a particular period. In the 
case of fixing risk for particular commodities or pests, this means determining an acceptable level of PR or PRC. Combining all of these options creates a matrix of choices for a regulatory authority (Figure 6).

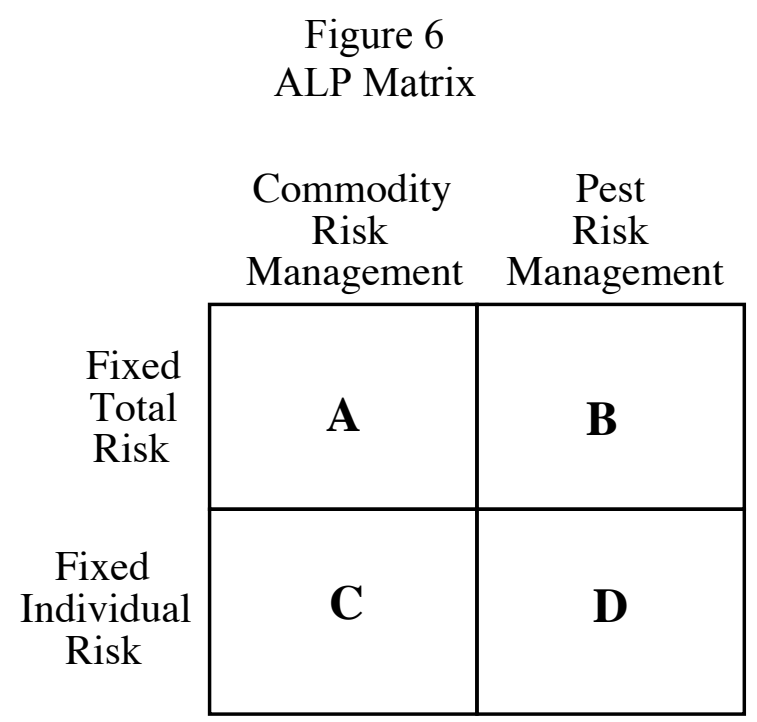

There are two different management options available for fixing total risk. A commodity-based management regime to fix total risk is shown by Box A in Figure 6. In this strategy, the regulator fixes the total acceptable risk over all trade, and then manages that risk by varying the acceptable risk for each commodity traded. In terms of the iso-risk framework, this is equivalent to determining a maximum sum for PRC over all $n$ commodities traded.

$$
\text { Total Risk }=\sum_{i=1}^{n} \mathrm{PRC}_{\mathrm{i}}
$$

Management of total risk is then done by managing PRC for each commodity. This is typically done by altering the likelihood of introduction. In order to stay within Total Risk, as trade volume increases or new commodities are introduced, more stringent phytosanitary measures would be required for some or all commodities. This could be done through changing any of the many factors influencing introduction potential, including trade volumes.

A pest-based management regime to fix total risk is shown by Box B in Figure 6 . In this strategy, the regulator fixes the total acceptable risk over all trade, and then manages that risk by varying the acceptable risk for each potential pest. In terms of the iso-risk framework, this is equivalent to determining a maximum sum for PR over all $n$ potential pests.

$$
\text { Total Risk }=\sum_{i=1}^{n} \mathrm{PR}_{\mathrm{i}}
$$

Management of total risk is then done by managing PR for each potential pest. This again is typically done by altering the likelihood of introduction.

In contrast to the Fixed Total Risk, Fixed Individual Risk means that the risk presented by any particular potential pest or commodity will be fixed, and the Total Risk will be variable, fluctuating with the level of 
trade. A commodity-based management regime to fix individual risk is shown by Box C in Figure 6 . In this strategy, the regulator fixes the individual acceptable risk for a commodity, and then manages the risk directly for each commodity traded. In terms of the iso-risk framework, this is equivalent to determining PRC for each commodity traded, following the process discussed earlier. A pest-based management regime to fix individual risk is shown by Box D in Figure 6. In this strategy, the regulator fixes the individual acceptable risk for a commodity, and then manages the risk directly for each pest. In terms of the iso-risk framework, this is equivalent to determining PR for each potential pest, following the process discussed earlier. In either case, total risk increases with the volume of trade.

In essence, fixing total risk means that individual risk is variable. This complicates management since management efforts must change with changes to trade volumes. This can be done either by modifying the risk accepted for the marginal product traded, leaving the risk accepted for earlier volumes at a higher level, or by modifying the risk of all products imported. In a practical sense, fixing total risk (either A or B) is likely to be an inappropriate basis for developing phytosanitary standards.

\section{Summary}

This paper has presented a methodology, Iso-Risk, for quantifying technical trade barriers that contain elements of risk of occurrence and economic impacts. The method provides a means for creating benchmarks and comparing quarantine treatments. This provides an important change from previous practice in that both economic and scientific criteria can be included in an analysis. This ensures that barriers can be treated on the basis of expected outcome rather than the technical characteristics of the barrier. As such, it is possible to move beyond simple considerations of whether the barrier involves an insect or a bacteria, and instead focus on whether a potential event behind the barrier is above, below or within an expected dollar value.

The Iso-Risk framework provides a solution to some of the problems created by SPS in a trade environment. In particular it allows for the even treatment of technical barriers and satisfies the need for transparent and measurable criteria for justifying decisions to trading partners. Using Iso-Risk, equivalent treatment requires that technical barriers or SPS have similar outcomes. This means that two exporters can be subjected to different quarantine requirements, but not violate WTO rules on equal treatment since the outcomes of the measures are similar. Justification of quarantine measures also becomes easier since decisions can be shown to be consistent within an overall domestic policy context.

Development of standards for objectively comparing quarantine measures is going to require some consensus on the appropriate economic impact to measure, the appropriate calculation of risk of establishment, and the appropriate ALP. Initially, a country would only be able to determine whether from an internal perspective it is treating its trading partners consistently using domestic definitions of economic impact and probability of establishment. This internal consistency of quarantine policy would be relative to a domestic ALP. At a later stage, when a number of countries were basing decisions on IsoRisk, it is possible that an international norm for ALP would emerge. A country could then establish, or perhaps be challenged, as to whether its treatment of trading partners was consistent with international norms.

The problem of arriving at an ALP which adequately describes a regulatory agency's perception of acceptable Pest Risk in an Iso-Risk framework can be approached by starting with a country's current regulatory treatment of pests and commodities. To establish an ALP, a sufficient sample of pests would first need to be evaluated for probability of entry and potential economic impacts after post-quarantine treatment. ALP should emerge from the pattern of plotted results, being represented by a line above which there would be no plots. An ALP for commodities could be determined by a similar process. A value for ALP implicit in existing quarantine regulations should emerge from the analysis. The process is not likely to be easy in practice since such an analysis may show inconsistencies in existing quarantine policies based on the resulting values of commodity and pest risk. 
While providing a clearer picture of ALP, experience in New Zealand has shown that there is a significant increase in information and analysis required by a quarantine authority when it has to include economic impact assessment and a specific probability of introduction. In many cases, little will be know about the economics of particular crops, much less the expected economic impact on a particular plant or probabilities of introduction. In addition to the problem of basic data, there is a problem with producing a rapid analysis for quarantine decisions if the level of detail implied by Iso-Risk is required for each commodity that is traded. Models to facilitate rapid analysis have been developed for NZMAF that calculate probability of introduction (Greer and Bigsby 1995; Greer et al 1995) and economic impacts (Bigsby and Crequer 1995; Bigsby 1995) based a standardised set of factors.

\section{References}

Bigsby, H.R. (1996). Enhancement and Further Development of the Economic Impact Assessment Modules. Final Report for MAF.

Bigsby, H.R. and Crequer, J. (1995). Pest Plant Economic Impact Assessment Model. Prepared for MAF Regulatory Authority, Wellington.

Bigsby, H.R. (1995). Pest Model - Partial Equilibrium Model User's Manual. AERU Report for the Ministry of Agriculture and Fisheries, Wellington.

Bigsby, H.R. and Crequer, J. (1998). Conceptual model for the management of pest risk. In, Fletcher, D.J., Kavalieris, L. and Manly, B.F.J. (Eds). Statistics in Ecology and Environmental Monitoring 2. Otago Conference Series 6, University of Otago Press.

Bigsby, H.R. and Whyte, C.F. (2000). Quantifying Phytosanitary Barriers to Trade. In, Hooker, N. and. Murano, E. (Eds.), Interdisciplinary Food Safety Research, CRC Press (in publication).

Bigsby, H.R. and Whyte, C (1998). A Model of the Appropriate Level of Protection for New Zealand's Quarantine Security. MAF Policy Draft Technical Paper, Wellington.

Cowley, J.M., Baker, R.T., Hill, C. F. and Barber, C.J. (1993). Pest Risk Assessment, Mediterranean fruit fly (Ceratitis capitata). Report of the PRA Committee, NZ Plant Protection Centre - Lynfield, to MAF Regulatory Authority.

Cohrssen, J.J. and Covello, V.T. (1989). Risk analysis: a guide to principles and methods for analyzing health and environmental risks. United States Council on Environmental Quality, Executive Office of the President.

DPIE (1997). Salmon Import Risk Analysis. Department of Primary Industries, Canberra.

FAO (1996). International Standards for Phytosanitary Measures. International Plant Protection Convention. FAO, Rome.

Firko. M.J. and Podleckis, E.V. (1996). Importation of Purple Passion Fruit (Passiflora Edulis) from Chile into the United States. USDA-APHIS, Riverdale MD.

Greer, G. and Bigsby, H. (1995). Estimating the economic impacts of pest introduction. Report of the Agribusiness and Economics Research Unit, Lincoln University, to MAF Policy.

Greer G., Bigsby, H. and McAuliffe, R. (1995). Pest Model - Partial Budget Model User's Manual. AERU Report for the Ministry of Agriculture and Fisheries, Wellington.

Hillman, J. (1991). Technical Barriers to Agricultural Trade. Westview Press, Boulder. 
Hillman, J. (1978). Nontariff Agricultural Trade Barriers. University of Nebraska Press, Lincoln.

James, S. and Anderson, K. (1998). On the need for more economic assessment of quarantine policies. Aust. J. Agric. Res. Econ. 42:4, pp. 425-444.

Kaplan, S. and Garrick, B. J. (1981). On the quantitative definition of risk. Risk Analysis 1, pp. 11-27.

Miller, L., McElvaine, M.D. R., McDowell, M. and Ahl, A. S. (1993). Developing a quantitative risk assessment process. Rev. Sci. Tech. Off. Int. Epiz. 12, pp. 1153-1164.

Ministry of Health (1996). Managing risks - a concept paper. Report of the Food and Nutrition Section, Public Health Group, of the New Zealand Ministry of Health.

Ndayisenga, F. and Kinsey, J. (1994). The structure of non-tariff trade measures on agricultural products in high-income countries. Agribusiness 10:4, pp.275-292.

Orr, R.L. (1995). A plant quarantine risk-based framework for standardising the acceptable level of risk and appropriate level of protection. Animal and Plant Health Inspection Service, USDA.

Papasolomontos, A. (1993). Present global activities in the harmonization of plant quarantine. In International Approaches to Plant Pest Risk Analysis. Proceedings of the APHIS/NAPPO International Workshop on the Identification, Assessment and Management of Risks due to Exotic Agricultural Pests. Alexandria, Virginia. NAPPO Bulletin 11.

Patterson, E. (1990). International efforts to minimize the adverse trade effects of national sanitary and phytosanitary regulations. J. World Trade 24:2, pp. 91-102.

Roberts, D., Josling, T. and Orden, D. (1999). A Framework for Analysing Technical Trade Barriers in Agricultural Markets. USDA-ERS Tech. Bull. 1876. Washington, D.C.

Smith, J.R. (1993). Welcoming Remarks. In International Approaches to Plant Pest Risk Analysis. Proceedings of the APHIS/NAPPO International Workshop on the Identification, Assessment and Management of Risks due to Exotic Agricultural Pests. Alexandria, Virginia. NAPPO Bulletin 11.

USDA (1996). Pathway-Initiated Pest Risk Assessment: Guidelines for Qualitative Assessments, version 4.0. USDA-APHIS-PPQ, Riverdale, MD.

USDA (1991). Pest Risk Assessment on the Improtation of Larch from Siberia and the Soviet Far East. USDA-Forest Service, Misc. Pub. 1495. Washington, D.C.

Whyte, C.F. (1998). Appropriate Levels of Protection: Equivalence of Commercial and Non-Commercial Pathways. Paper presented at the Pre-conference Workshop on Border Security and Risk Analysis, January 19, 1999. Lincoln University.

Whyte, C.F., Baker, R.T. and Cowley, J.M. (1998). Procedures for plant pest risk assessment: biological risk assessment for Mediterranean fruit fly. In, Fletcher, D.J., Kavalieris, L. and Manly, B.F.J. (Eds). Statistics in Ecology and Environmental Monitoring 2. Otago Conference Series 6, University of Otago Press.

Whyte, C.F. and Cowley, J.M. (1996). Effect of x-ray machines on the risk of fruit fly outbreaks in New Zealand. B. Assessment of the annual probability of fruit fly outbreaks in New Zealand from passenger baggage. Report to MAF Policy. 
Whyte, C.F., Cowley, J.M., Baker, R.T., Hill, C.F., Barber, C.J. and Braithwaite, M. (1995). Economic risk assessment, Mediterranean fruit fly (Ceratitis capitata). Report of the PRA Committee of the NZ Plant Protection Centre for MAF Regulatory Authority. 